\title{
Tri(pyridinyl)pyridine Viologen-based Kagome Dual Coordination Polymer with Selective Chromic Response to Soft X-ray and Volatile Organic Amines
}

Shi-Li Li, * Mei Li, Yan Zhang, Hui-Min Xu and Xian-Ming Zhang*

Key Laboratory of Magnetic Molecules and Magnetic Information Materials (Ministry of Education), School of Chemistry \& Material Science, Shanxi Normal University, Linfen 041004, P. R. China 


\section{General Method}

The solid-state UV/vis absorption spectra were determined on a TU-1901 spectrophotometer with an IS19-1 integrating sphere in the wavelength range of $200-$ $850 \mathrm{~nm}$. Electron paramagnetic resonance (EPR) spectra were obtained on a Bruker EMX spectrometer. Powder X-ray diffraction (PXRD) patterns were tested with a Rigaku MiniFlex II X-ray diffractometer using $\mathrm{Cu} \mathrm{K \alpha}$ radiation $(\lambda=1.5418 \AA, 2 \theta$ range from $4^{\circ}$ to $40^{\circ}$ ) at $293 \mathrm{~K}$. Thermogravimetric (TG) data were carried out using a SETARAM LABSYS equipment at a heating rate of $10{ }^{\circ} \mathrm{C} \min ^{-1}$ under nitrogen gas flow in the range of $30-800{ }^{\circ} \mathrm{C}$. Elemental analyses were performed on a vario EL-II analyzer. Fourier transform infrared (FT-IR) spectra were recorded the range of 4000-400 $\mathrm{cm}^{-1}$ using a Nicolet 5DX spectrometer with $\mathrm{KBr}$ pellets. X-ray photoelectron spectroscopy was recorded by a Thermo Scientific K-Alpha X-ray photoelectron spectrometer (XPS).

Table S1. Selected bond lengths $[\AA]$ and angles [deg] for $\mathbf{1}$.

\begin{tabular}{llll}
\hline $\mathrm{Mn}(1)-\mathrm{O}(6 \mathrm{a})$ & $2.107(3)$ & $\mathrm{Mn}(2)-\mathrm{O}(2)$ & $2.168(3)$ \\
$\mathrm{Mn}(1)-\mathrm{O}(1)$ & $2.117(4)$ & $\mathrm{Mn}(2)-\mathrm{O}(2 \mathrm{c})$ & $2.168(3)$ \\
$\mathrm{Mn}(1)-\mathrm{O}(4 \mathrm{~b})$ & $2.224(3)$ & $\mathrm{Mn}(2)-\mathrm{O}(5 \mathrm{a})$ & $2.170(3)$ \\
$\mathrm{Mn}(1)-\mathrm{O}(3 \mathrm{~b})$ & $2.445(4)$ & $\mathrm{Mn}(2)-\mathrm{O}(5 \mathrm{~d})$ & $2.170(3)$ \\
$\mathrm{Mn}(1)-\mathrm{Cl}(2)$ & $2.4784(17)$ & $\mathrm{Mn}(2)-\mathrm{O}(3 \mathrm{e})$ & $2.228(3)$ \\
$\mathrm{Mn}(1)-\mathrm{Cl}(1)$ & $2.5325(14)$ & $\mathrm{Mn}(2)-\mathrm{O}(3 \mathrm{~b})$ & $2.228(3)$ \\
$\mathrm{O}(6 \mathrm{a})-\mathrm{Mn}(1)-\mathrm{O}(1)$ & $90.86(18)$ & $\mathrm{O}(2)-\mathrm{Mn}(2)-\mathrm{O}(2 \mathrm{c})$ & 180.0 \\
$\mathrm{O}(6 \mathrm{a})-\mathrm{Mn}(1)-\mathrm{O}(4 \mathrm{~b})$ & $149.92(16)$ & $\mathrm{O}(2)-\mathrm{Mn}(2)-\mathrm{O}(5 \mathrm{a})$ & $94.42(14)$ \\
\hline
\end{tabular}




\begin{tabular}{lcll}
\hline $\mathrm{O}(1)-\mathrm{Mn}(1)-\mathrm{O}(4 \mathrm{~b})$ & $91.89(19)$ & $\mathrm{O}(2 \mathrm{c})-\mathrm{Mn}(2)-\mathrm{O}(5 \mathrm{a})$ & $85.58(14)$ \\
$\mathrm{O}(6 \mathrm{a})-\mathrm{Mn}(1)-\mathrm{O}(3 \mathrm{~b})$ & $93.19(13)$ & $\mathrm{O}(2)-\mathrm{Mn}(2)-\mathrm{O}(5 \mathrm{~d})$ & $85.58(14)$ \\
$\mathrm{O}(1)-\mathrm{Mn}(1)-\mathrm{O}(3 \mathrm{~b})$ & $89.56(16)$ & $\mathrm{O}(2 \mathrm{c})-\mathrm{Mn}(2)-\mathrm{O}(5 \mathrm{~d})$ & $94.42(14)$ \\
$\mathrm{O}(4 \mathrm{~b})-\mathrm{Mn}(1)-\mathrm{O}(3 \mathrm{~b})$ & $56.90(13)$ & $\mathrm{O}(5 \mathrm{a})-\mathrm{Mn}(2)-\mathrm{O}(5 \mathrm{~d})$ & $180.00(19)$ \\
$\mathrm{O}(6 \mathrm{a})-\mathrm{Mn}(1)-\mathrm{Cl}(2)$ & $106.79(12)$ & $\mathrm{O}(2)-\mathrm{Mn}(2)-\mathrm{O}(3 \mathrm{e})$ & $83.19(14)$ \\
$\mathrm{O}(1)-\mathrm{Mn}(1)-\mathrm{Cl}(2)$ & $84.82(14)$ & $\mathrm{O}(2 \mathrm{c})-\mathrm{Mn}(2)-\mathrm{O}(3 \mathrm{e})$ & $96.81(14)$ \\
$\mathrm{O}(4 \mathrm{~b})-\mathrm{Mn}(1)-\mathrm{Cl}(2)$ & $103.29(11)$ & $\mathrm{O}(5 \mathrm{a})-\mathrm{Mn}(2)-\mathrm{O}(3 \mathrm{e})$ & $88.55(12)$ \\
$\mathrm{O}(3 \mathrm{~b})-\mathrm{Mn}(1)-\mathrm{Cl}(2)$ & $159.28(8)$ & $\mathrm{O}(5 \mathrm{~d})-\mathrm{Mn}(2)-\mathrm{O}(3 \mathrm{e})$ & $91.45(12)$ \\
$\mathrm{O}(6 \mathrm{a})-\mathrm{Mn}(1)-\mathrm{Cl}(1)$ & $88.78(11)$ & $\mathrm{O}(2)-\mathrm{Mn}(2)-\mathrm{O}(3 \mathrm{~b})$ & $96.81(14)$ \\
$\mathrm{O}(1)-\mathrm{Mn}(1)-\mathrm{Cl}(1)$ & $179.12(15)$ & $\mathrm{O}(2 \mathrm{c})-\mathrm{Mn}(2)-\mathrm{O}(3 \mathrm{~b})$ & $83.19(14)$ \\
$\mathrm{O}(4 \mathrm{~b})-\mathrm{Mn}(1)-\mathrm{Cl}(1)$ & $88.01(11)$ & $\mathrm{O}(5 \mathrm{a})-\mathrm{Mn}(2)-\mathrm{O}(3 \mathrm{~b})$ & $91.45(12)$ \\
$\mathrm{O}(3 \mathrm{~b})-\mathrm{Mn}(1)-\mathrm{Cl}(1)$ & $89.66(8)$ & $\mathrm{O}(5 \mathrm{~d})-\mathrm{Mn}(2)-\mathrm{O}(3 \mathrm{~b})$ & $88.55(12)$ \\
$\mathrm{Cl}(2)-\mathrm{Mn}(1)-\mathrm{Cl}(1)$ & $96.05(6)$ & $\mathrm{O}(3 \mathrm{e})-\mathrm{Mn}(2)-\mathrm{O}(3 \mathrm{~b})$ & $180.00(16)$ \\
\hline
\end{tabular}

Symmetry code: a) $-x+2,-y+1,-z+1$; b) $-x+3 / 2, y-1 / 2,-z+1$; c) $x-1, y, z ; d)-x+1,-y+1$, $-z+1$; e) $x-1 / 2,-y+3 / 2, z ; f)-x+3 / 2, y+1 / 2,-z+1 ; g) x+1, y, z$. 


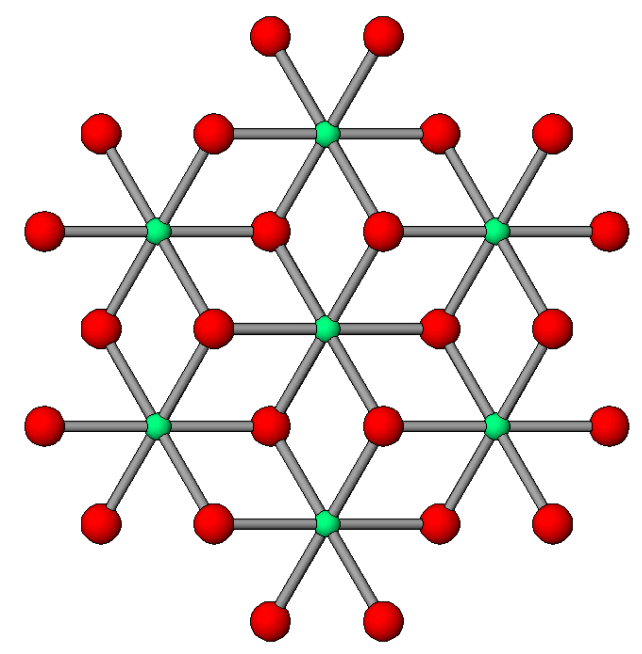

Figure S1. Simplified 2D Kagome Dual layer of 1. Green and red balls represent six-connected trinuclear $\left[\mathrm{Mn}_{3}\left(\mathrm{CO}_{2}\right)_{6} \mathrm{Cl}_{4}\right]$ units and three-connected tpptb ligands, respectively.

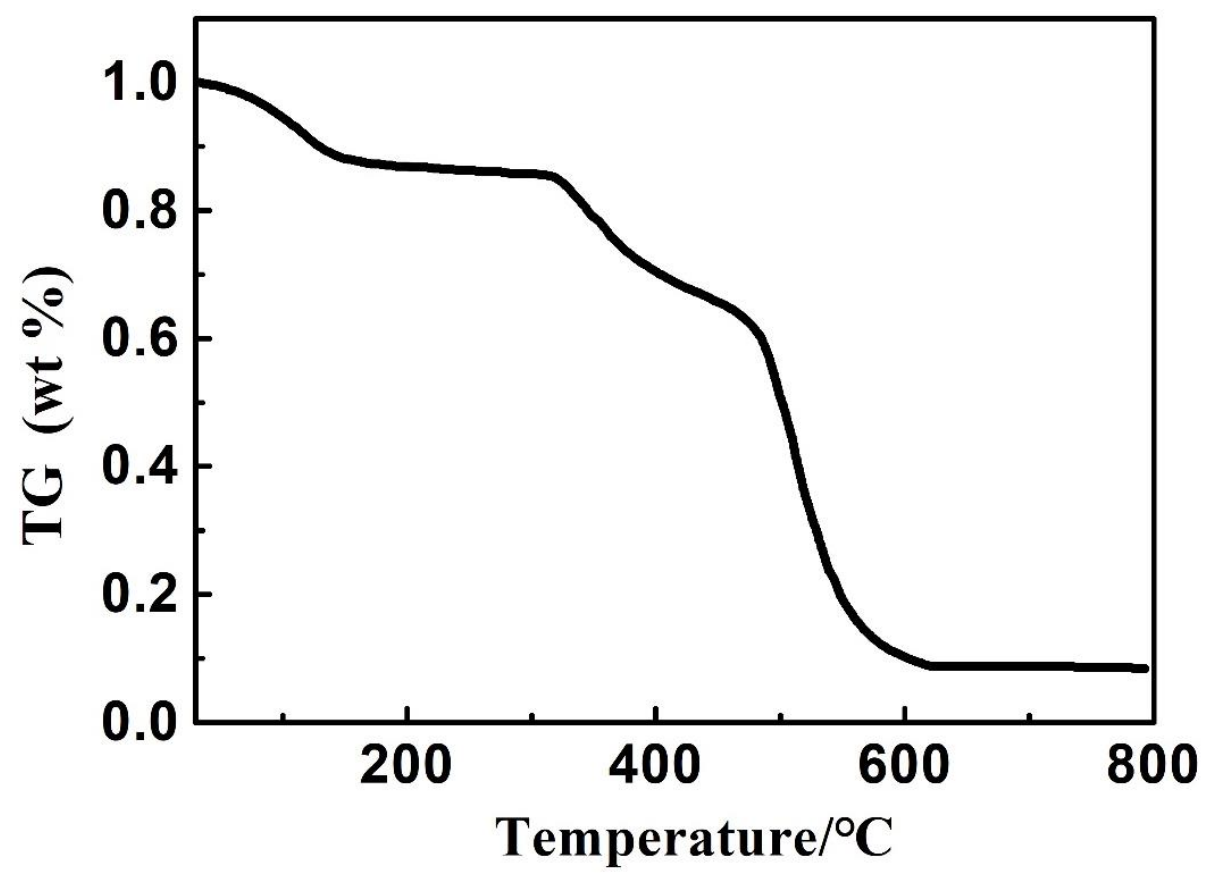

Figure S2. The TG plots for $\mathbf{1}$ under nitrogen atmosphere at heating rate of $10{ }^{\circ} \mathrm{C} / \mathrm{min}$. 


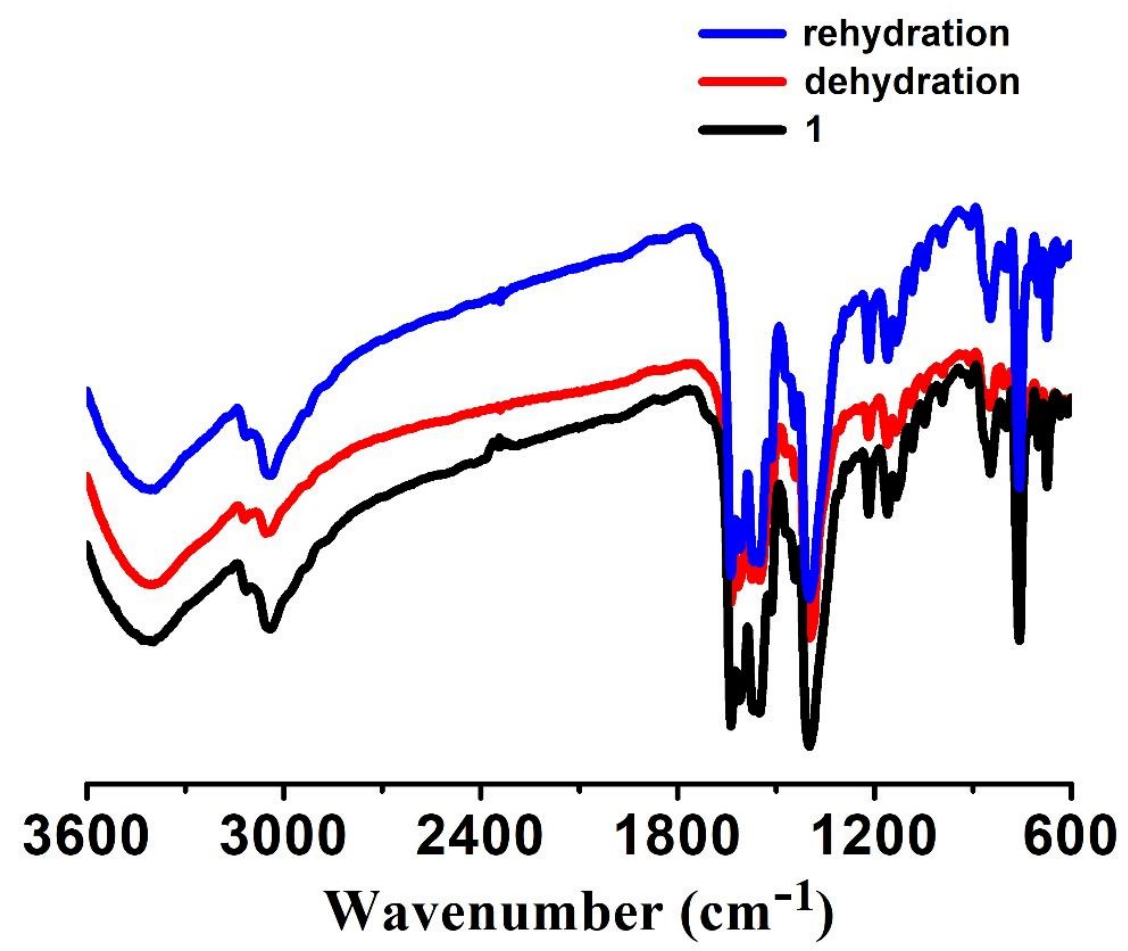

Figure S3. IR spectra of 1, 1-dehydration and 1-rehydration.

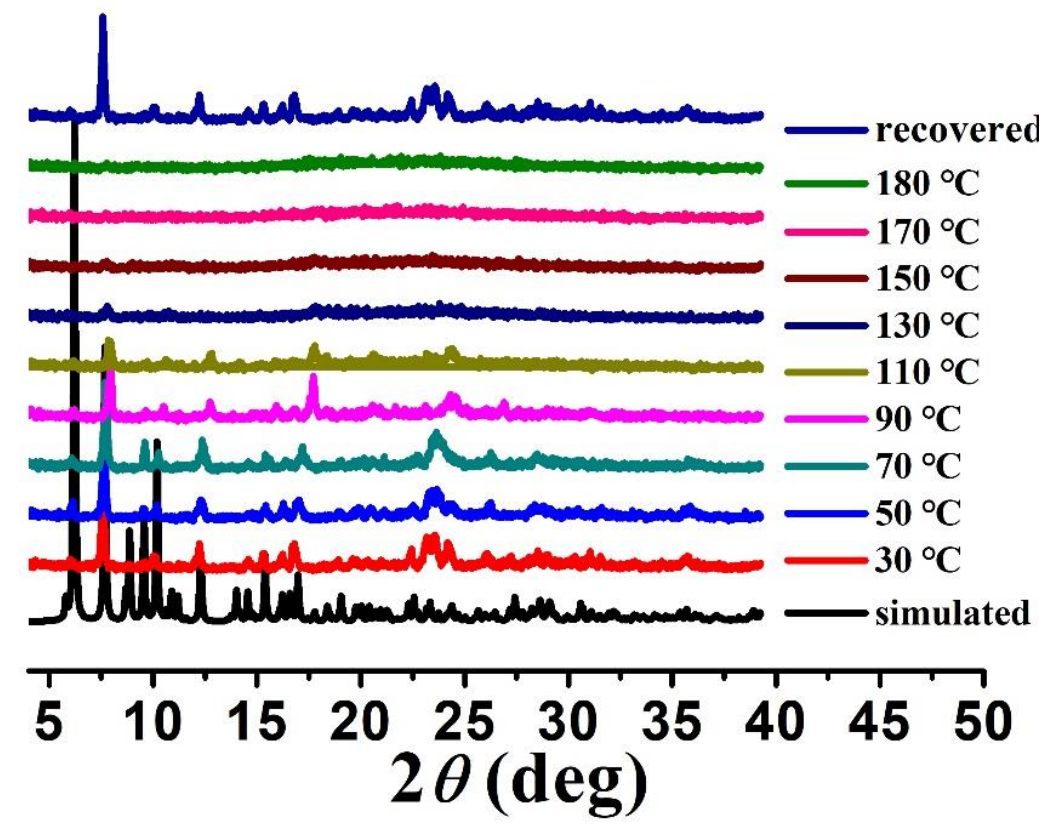

Figure S4. PXRD profiles of $\mathbf{1}$ heated at different temperature. 


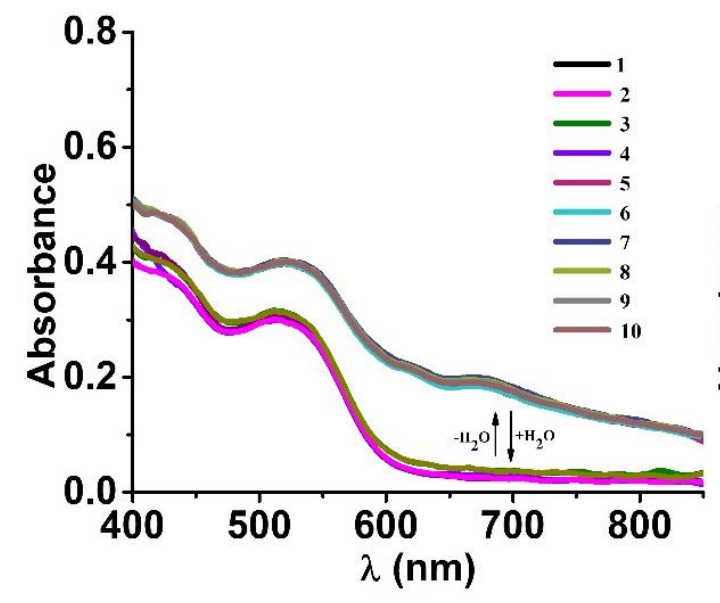

(a)

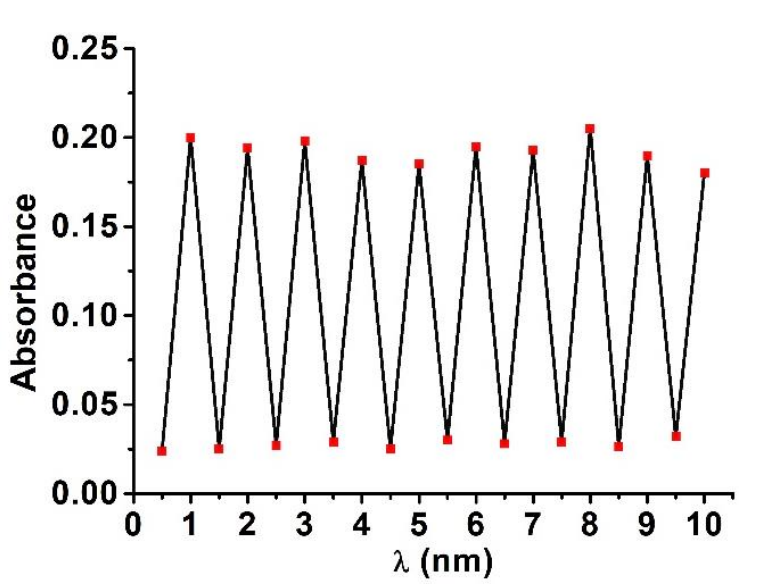

(b)

Figure S5. UV-vis diffuse-reflectance spectra (a) A plot of the absorption at $677 \mathrm{~nm}$ versus the number of cycles (b) for the reversible hydrochromic behavior of $\mathbf{1}$.

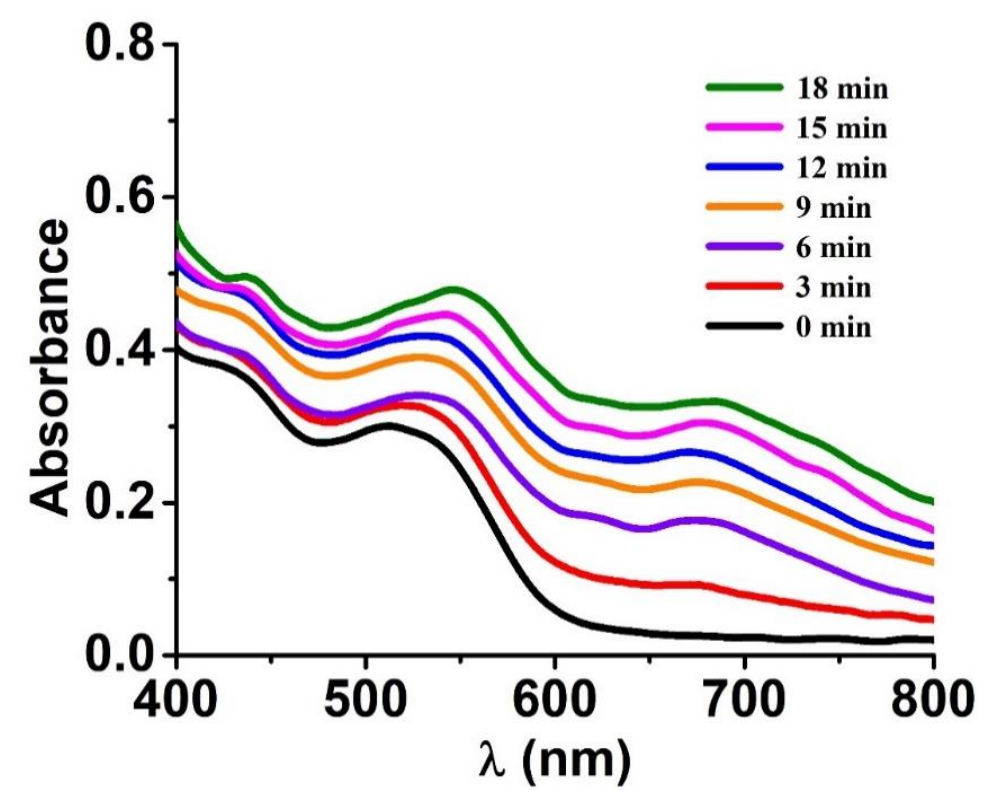

Figure S6. Time-dependent UV-vis spectra of 1 upon heating at $90{ }^{\circ} \mathrm{C}$.

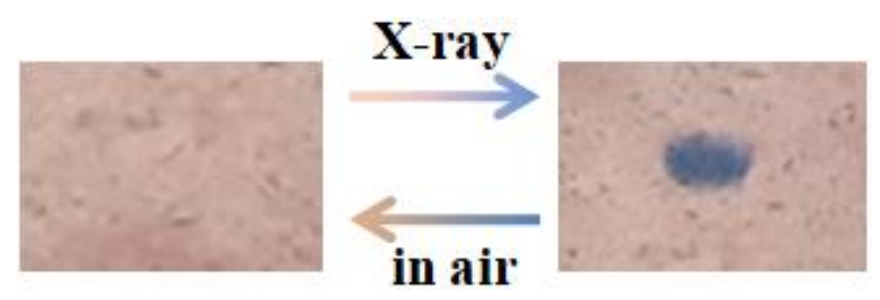

Figure S7. Soft X-ray-induced color change was captured using an X-ray photoelectron spectrometer (Al-Ka, $\lambda=8.357 \AA$ ) after illumination for about several 
minutes.

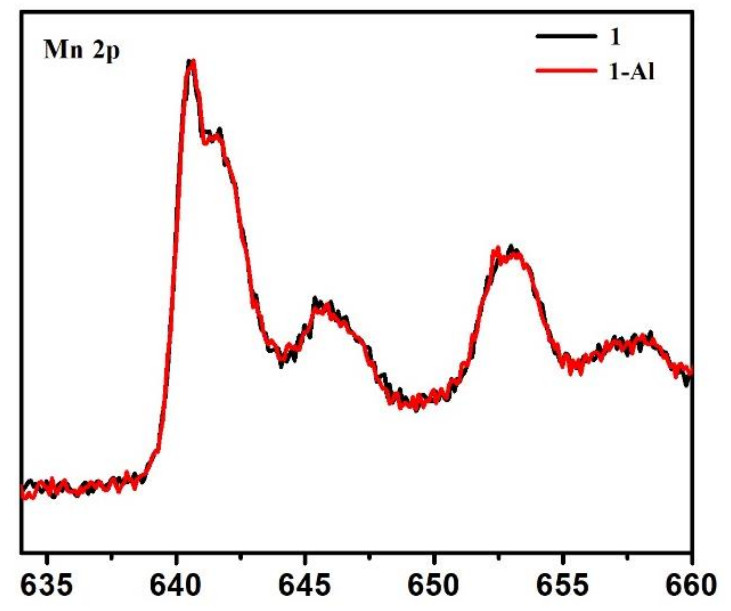

Binding Energy / eV
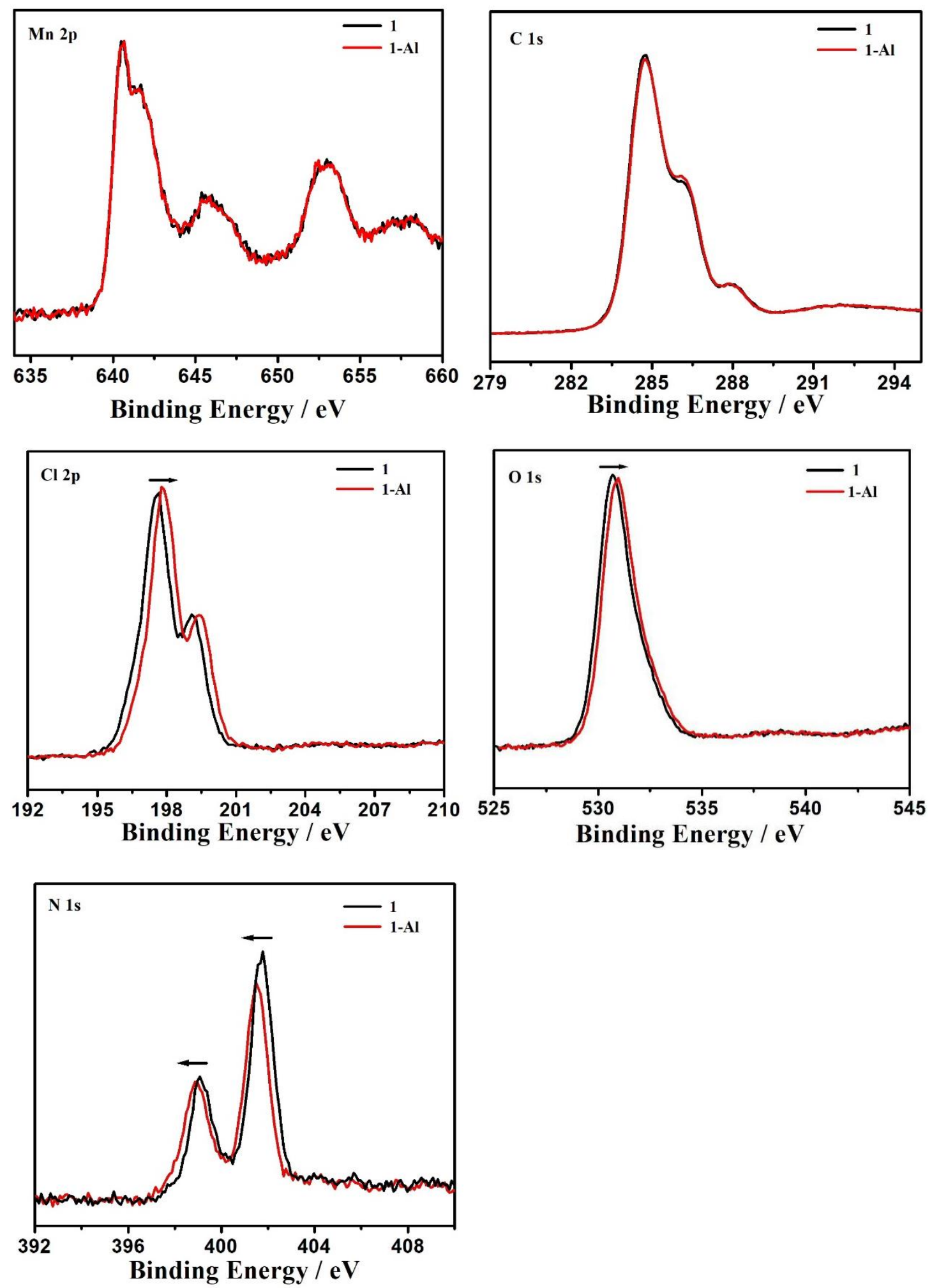

Figure S8. XPS spectra of $\mathbf{1}$ and $\mathbf{1}$-Al. 




Figure S9. The $\mathrm{C}-\mathrm{H} \cdots \mathrm{O}$ and $\mathrm{C}-\mathrm{H} \cdots \mathrm{Cl}$ distances between electron acceptor and donor.

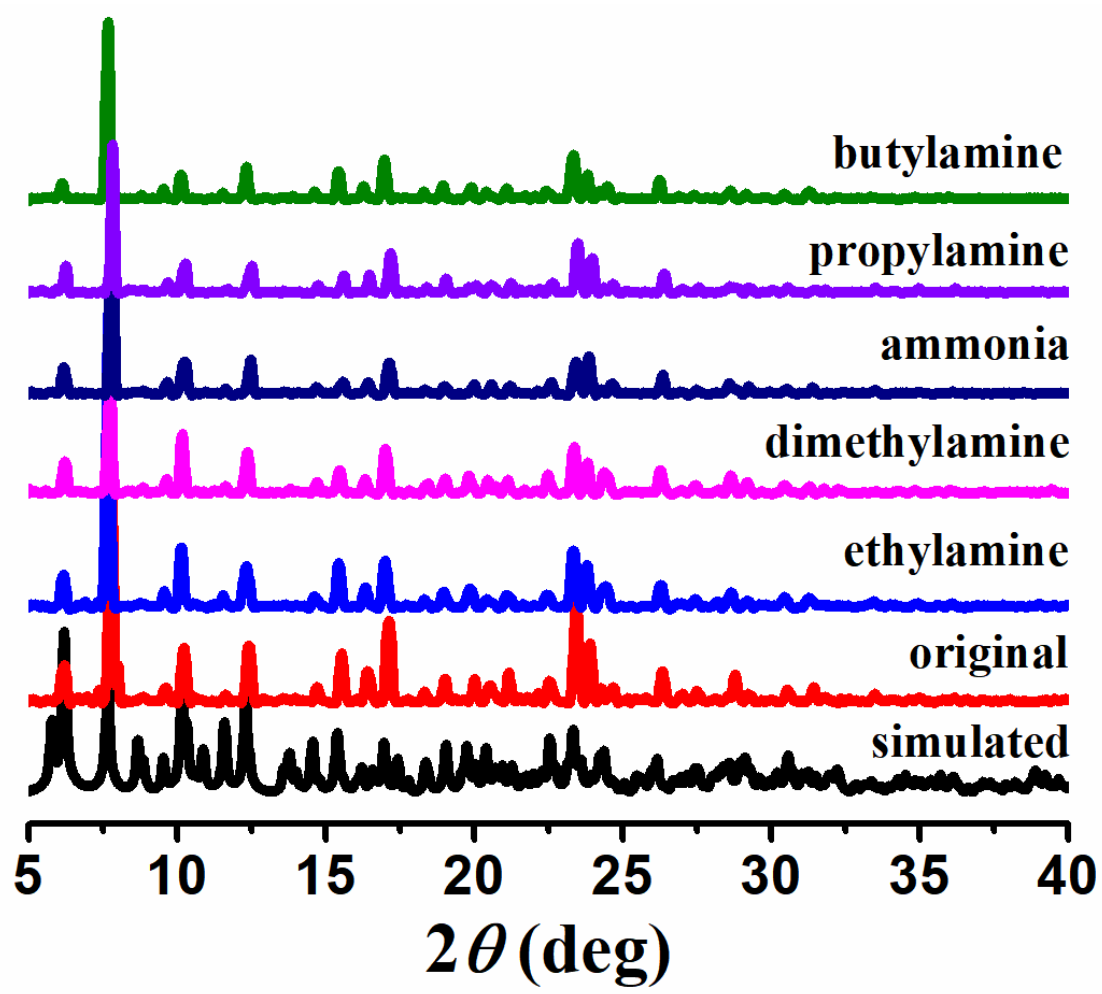

Figure S10. PXRD profiles of 1 fumigated with various organic amine vapors. 